\title{
Data-driven Gradient-based Point-to-Point Iterative Learning Control for Non-linear Systems
}

\author{
Benyan Huo · Chris T. Freeman • Yanghong Liu
}

Received: date / Accepted: date

\begin{abstract}
Iterative learning control (ILC) is a wellestablished methodology which has proven successful in achieving accurate tracking control for repeated tasks. However, the majority of ILC algorithms require a nominal plant model and are sensitive to modelling mismatch. This paper focuses on the class of gradientbased ILC algorithms and proposes a data-driven ILC implementation applicable to a general class of nonlinear systems, in which an explicit model of the plant dynamics is not required. The update of the control signal is generated by an additional experiment executed between ILC trials. The framework is further extended by allowing only specific reference points to be tracked, thereby enabling faster convergence. Necessary convergence conditions and corresponding convergence rates for both approaches are derived theoretically. The proposed data-driven approaches are demonstrated through application to a stroke rehabilitation problem requiring accurate control of nonlinear artificiallystimulated muscle dynamics.
\end{abstract}

Keywords Iterative learning control · Data-driven control · Nonlinear systems · Point-to-point tracking . Stroke rehabilitation

This study was funded by National Natural Science Foundation of China (NO.61473265), the China Postdoctoral Science Foundation (NO.2018M632801) and the ZZU-Southampton Collaborative Research Project under Grant 16306/01. Part of the results in this paper were submitted to 13th IFAC Workshop on Adaptive and Learning Control Systems [22].

B. Huo $\cdot$ Y. Liu $(\bowtie)$

School of Electrical Engineering, Zhengzhou University, 100

Science Avenue, Zhengzhou, Henan, 450001 China

E-mail: liuyh@zzu.edu.cn

C. T. Freeman

School of Electronics and Computer Science, University of Southampton, Southampton, UK, SO17 1BJ

\section{Introduction}

Iterative learning control (ILC) is a control paradigm which enables high-performance tracking of tasks which are executed repeatedly over a time interval, each attempt termed a 'trial'. Distinct from traditional feedback control, standard ILC algorithms can be regarded as implementations of open-loop feedforward control, in which the historical set of tracking error sequences are used to modify the control signal for the next trial [29]. The tracking error over the finite time interval has potential to converge to zero after sufficient trials. Although initially proposed as a model-free approach [3], practical application has been limited by difficulties in tuning control parameters and sensitivity to noise and uncertainty. As a specific class of modelbased ILC, gradient based algorithms have drawn considerable attention in both theoretical and application domains due to their well-defined convergence and robustness properties [30]. This class includes gradient ILC $[34,31]$, inverse ILC [32] and norm optimal ILC [9, $1]$, which have been applied to diverse applications including gantry robots [35], multi-axis robotic testbeds [5], rehabilitation platforms [36] and pneumatic muscle actuators [39].

Gradient-based ILC approaches have been applied to nonlinear systems, following linearization via feedback linearization [7], input-output linearization [18], and piecewise linearization [2]. Newton-based ILC algorithms were also proposed for a class of nonlinear system in $[24,40]$ based on linearizing the system about an operating point which was modified for each ILC trial. In traditional ILC, the system is required to track the given reference trajectory at all sample times. However, in some applications only a few prescribed points need to be considered, such as point-to-point rehabilita- 
tion movements [33] and 'pick and place' robotic tasks [11]. To address this need, point-to-point ILC methods have been proposed in $[19,14]$, and have proven effective in improving control performance (e.g. reducing control effort, increasing convergence speed and reducing computational load).

The aforementioned ILC approaches require an accurate plant model to achieve rapid and monotonic convergence, and their tolerance to model uncertainty is limited [30]. This is reflected in applications where control performance has significantly deteriorated for systems with complex dynamics, coupled dynamics and multivariate, nonlinear or time-varying systems [15]. To address these cases, data-driven ILC control approaches were proposed, in which the control signal is updated by the input and output data without explicit plant information. These approaches can be classified into two categories: system approximation methods and additional experiment methods. In system approximation methods, input and output data are used to approximate system dynamics via Fourier series [26], neutral networks [25], fuzzy basis functions [13], dynamic linearization data models [12], or finite impulse response filters [23]. Although such methods do not require a model, the approximate precision of the system dynamics affects the control performance. The second approach updates the control signal directly using outputs of extra experiments designed to capture the system characteristics over a region of interest. This concept was first proposed for ILC in [17], and subsequently [10] used additional experiments to estimate the gradient and proved that the estimation is unbiased in the presence of noise. This data-driven approach was then extended to multivariate linear systems in [6] and its convergence conditions and connections to other common ILC forms were derived.

In this paper, we propose a new data-driven gradientbased ILC framework suitable for nonlinear systems and further extend the result to point-to-point tasks to improve the convergence rate and design flexibility. The main contributions of this paper are:

1. a new data-driven approach for nonlinear systems is proposed in Section 3 based on the additional experiment method. Conditions to guarantee convergence to zero error are derived, as well as bounds on the convergence rate. Distinct from the online approximation methods of $[26,25,13,12,23]$, there is no need to estimate a data model and the control signals are updated using the outputs of additional experiments directly.

2 . the proposed approach is further extended to pointto-point tasks in Section 5. Distinct from traditional ILC algorithms, only prescribed points are tracked.
It is proved that, as the number of tracked points is reduced, the convergence rate increases.

3. the proposed approaches are verified by application to a rehabilitation problem involving a time-varying nonlinear electrically stimulated upper limb system in Section 6.

The paper is arranged as follows. The next section extends gradient ILC for use with a general class of nonlinear system, with Section 3 then showing how the required update can be generated in a model-free manner. Convergence conditions and bounds are derived in Section 4, and the framework is extended in Section 5 to support 'point-to-point' tracking at only a subset of reference points. In Section 6 the proposed approaches are applied to a rehabilitation problem, with conclusions then given in Section 7.

The notation is standard: the set of real numbers is denoted $\mathbb{R}$, the set of natural numbers is denoted $\mathbb{N}$, and the norm of any column vector $x \in \mathbb{R}^{n}$ is given by $\|x\|=\sqrt{x^{\top} x}$. The minimum and maximum singular values of a matrix $X \in \mathbb{R}^{n \times n}$ are denoted $\underline{\sigma}(\cdot)$ and $\bar{\sigma}(\cdot)$ respectively. For a time-indexed signal $x(t) \in \mathbb{R}^{n}$, with $t=0,1, \cdots N-1$, bold is used to denote the so-called 'lifted' supervector $\boldsymbol{x}=\left[x(0)^{\top}, x(1)^{\top}, \cdots, x(N-1)^{\top}\right]^{\top}$.

\section{Gradient-based ILC for Nonlinear systems}

In this section, we construct the gradient iterative learning control algorithm for nonlinear systems.

Consider the following single-input-single-output (SISO) discrete time nonlinear system

$$
\begin{aligned}
x(i+1) & =f(x(i), u(i)), & & x(0)=x_{0}, \\
y(i) & =h(x(i), u(i)), & & i=0,1, \ldots, N-1
\end{aligned}
$$

where $x(i) \in \mathbb{R}^{n}, u(i)$ and $y(i)$ are the state vector, input and output respectively, $f(\cdot)$ and $h(\cdot)$ are nonlinear functions which are continuously differentiable, $i$ is sampled time index of a trial, $N$ is the trial length. Then, the output $y$ could be represented by the input $u$ series and the initial state $x(0)$

$$
\begin{aligned}
y(0) & =h(x(0), u(0))=g_{0}(x(0), u(0)) \\
y(1) & =h(x(1), u(1))=h(f(x(0), u(0)), u(1)) \\
:= & g_{1}(x(0), u(0), u(1)) \\
& \vdots \\
y(N-1)= & h(x(N-1), u(N-1)) \\
= & h(f(x(N-2), u(N-2)), u(N-1)) \\
: & g_{N-1}(x(0), u(0), u(1), \ldots, u(N-1))
\end{aligned}
$$


so the system (1) can be transformed into a vector form

$\boldsymbol{y}=\left[\begin{array}{c}g_{0}(x(0), u(0)) \\ g_{1}(x(0), u(0), u(1)) \\ g_{2}(x(0), u(0), u(1), u(2)) \\ \vdots \\ g_{N-1}(x(0), u(0), u(1), \ldots, u(N-1))\end{array}\right]:=\boldsymbol{g}(\boldsymbol{u})$

where $\boldsymbol{u}=[u(0), u(1), \ldots, u(N-1)]^{\top} \in \mathbb{R}^{N}, \boldsymbol{y}=[y(0)$, $y(1), \ldots, y(N-1)]^{\top} \in \mathbb{R}^{N}$ and the dependence of $\boldsymbol{g}(\cdot)$ on $x(0)$ is not explicitly stated since it is not a controlled variable. The reference trajectory is similarly constructed as

$\boldsymbol{r}=[r(0), r(1), \ldots, r(N-1)]^{\top} \in \mathbb{R}^{N}$.

\subsection{ILC Problem Definition}

The ILC problem involves repeated trials at the tracking problem. After the $k^{\text {th }}$ trial system (1) is reset to initial state $x_{0}$. The next input $u_{k+1}$ is then formed using the previous error within update law

$\boldsymbol{u}_{k+1}=\boldsymbol{u}_{k}+\boldsymbol{l}\left(\boldsymbol{e}_{k}\right)$

where $\boldsymbol{l}(\cdot)$ is a vector function and the tracking error is $\boldsymbol{e}_{k}=\boldsymbol{r}-\boldsymbol{y}_{k}$. The aim is for convergence to the solutions

$\lim _{k \rightarrow \infty} \boldsymbol{u}_{k}=\boldsymbol{u}_{\infty}, \quad \quad \lim _{k \rightarrow \infty} \boldsymbol{e}_{k}=\mathbf{0}$

\subsection{Gradient-based ILC}

Within the ILC framework, the gradient descent method is applied to minimise the error norm on trial $k$,

$J\left(\boldsymbol{u}_{k}\right):=\left\|\boldsymbol{e}_{k}\right\|^{2}, \quad \boldsymbol{e}_{k}=\boldsymbol{r}-\boldsymbol{g}\left(\boldsymbol{u}_{k}\right)$,

subject to dynamics (1). As $\nabla J\left(\boldsymbol{u}_{k}\right)=-2 \nabla \boldsymbol{g}\left(\boldsymbol{u}_{k}\right)^{\top} \boldsymbol{e}_{k}$, the gradient ILC update law can be formulated as

$\boldsymbol{u}_{k+1}=\boldsymbol{u}_{k}+\beta \nabla \boldsymbol{g}\left(\boldsymbol{u}_{k}\right)^{\top} \boldsymbol{e}_{k}$

where scalar $\beta>0$ is the step length.

The term $\nabla \boldsymbol{g}\left(\boldsymbol{u}_{k}\right)$ can be regarded as the system linearization around an operating point $\boldsymbol{u}_{k}$. Without loss of generality, $\boldsymbol{u}_{k}$ is replaced by $\overline{\boldsymbol{u}}$. As shown in (3), the system is causal, thus $\nabla \boldsymbol{g}(\overline{\boldsymbol{u}})$ is represented as

$$
\begin{aligned}
\nabla \boldsymbol{g}(\overline{\boldsymbol{u}})= & {\left.\left[\begin{array}{cccc}
\frac{\partial g_{0}}{\partial u(0)} & \frac{\partial g_{0}}{\partial u(1)} & \cdots & \frac{\partial g_{0}}{\partial u(N-1)} \\
\frac{\partial g_{1}}{\partial u(0)} & \frac{\partial g_{1}}{\partial u(1)} & \cdots & \frac{\partial g_{1}}{\partial u(N-1)} \\
\vdots & \vdots & \ddots & \vdots \\
\frac{\partial g_{N-1}}{\partial u(0)} & \frac{\partial g_{N-1}}{\partial u(1)} & \cdots & \frac{\partial g_{N-1}}{\partial u(N-1)}
\end{array}\right]\right|_{\boldsymbol{u}=\overline{\boldsymbol{u}}} } \\
= & {\left.\left[\begin{array}{cccc}
\frac{\partial g_{0}}{\partial u(0)} & 0 & \cdots & 0 \\
\frac{\partial g_{1}}{\partial u(0)} & \frac{\partial g_{1}}{\partial u(1)} & \cdots & 0 \\
\vdots & \vdots & \ddots & \vdots \\
\frac{\partial g_{N-1}}{\partial u(0)} & \frac{\partial g_{N-1}}{\partial u(1)} & \cdots & \frac{\partial g_{N-1}}{\partial u(N-1)}
\end{array}\right]\right|_{\boldsymbol{u}=\overline{\boldsymbol{u}}} }
\end{aligned}
$$

Then, the linearized system $\boldsymbol{y}=\nabla \boldsymbol{g}(\overline{\boldsymbol{u}}) \boldsymbol{u}$ is equivalent to a linear time-varying (LTV) system

$$
\begin{aligned}
\boldsymbol{x}(i+1) & =A(i) \boldsymbol{x}(i)+B(i) u(i), \quad x(0)=x_{0}, \quad(9) \\
y(i) & =C(i) \boldsymbol{x}(i)+D(i) u(i), \quad i=0,1,2, \ldots, N-1
\end{aligned}
$$

where $A(i) \in \mathbb{R}^{n \times n}, B(i) \in \mathbb{R}^{n}, C(i) \in \mathbb{R}^{1 \times n}$, and $D(i) \in \mathbb{R}$. In particular,

$$
A(i)=\left.\frac{\partial f}{\partial x}\right|_{\begin{array}{l}
u=\bar{u}(i) \\
x=\bar{x}(i)
\end{array}}, B(i)=\left.\frac{\partial f}{\partial u}\right|_{\begin{array}{l}
u=\bar{u}(i) \\
x=\bar{x}(i)
\end{array}}
$$

$$
C(i)=\left.\frac{\partial h}{\partial x}\right|_{\begin{array}{l}
u=\bar{u}(i) \\
x=\bar{x}(i)
\end{array}}, D(i)=\left.\frac{\partial h}{\partial u}\right|_{\begin{array}{r}
u=\bar{u}(i) \\
x=\bar{x}(i)
\end{array}}
$$

in which $\overline{\boldsymbol{x}}$ is generated by $\bar{x}(i+1)=f(\bar{x}(i), \bar{u}(i))$, $\bar{x}(0)=x_{0}$. It follows that $\nabla \boldsymbol{g}(\overline{\boldsymbol{u}})=$

$$
\left[\begin{array}{cccc}
D(0) & 0 & \cdots & 0 \\
C(1) B(0) & D(1) & \cdots & 0 \\
\vdots & \vdots & \ddots & \vdots \\
N-2 & N-2 & & \\
C(N-1) \prod_{i=1}^{N-2} A(i) B(0) & C(N-1) \prod_{i=2}^{N} A(i) B(1) & \cdots & D(N-1)
\end{array}\right]
$$

which equates to (8) via the identity

$$
\left.\frac{\partial g_{i}}{\partial u(j)}\right|_{\boldsymbol{u}=\overline{\boldsymbol{u}}}= \begin{cases}C(i) \prod_{l=j+1}^{i-1} A(l) B(j), & j=0,1, \ldots i-1 \\ D(i), & j=i\end{cases}
$$




\section{Data-driven Gradient-based ILC for nonlinear systems}

To apply the data-driven approach proposed for linear systems in [17], the gradient ILC update law (7) has to be reformed as

$\boldsymbol{u}_{k+1}=\boldsymbol{u}_{k}+\beta \nabla \boldsymbol{g}\left(\hat{\boldsymbol{u}}_{k}\right)^{\top} \boldsymbol{e}_{k}$

where $\hat{\boldsymbol{u}}_{k}$ is a fixed operating point to be determined. One particular choice that will be justified by the convergence analysis in Section 4 is

$\hat{\boldsymbol{u}}_{k}(i)=\hat{u}_{k}, \quad \hat{u}_{k}:=\min _{\hat{u}_{k} \in \mathbb{R}}\left\|\boldsymbol{u}_{k}-\hat{\boldsymbol{u}}_{k}\right\|^{2}$

where scalar $\hat{u}_{k}$ is the average value of the control sequence $\hat{\boldsymbol{u}}_{k}$, i.e.

$\hat{u}_{k}=\frac{1}{N} \sum_{i=0}^{N-1} u_{k}(i)$,

with corresponding fixed $\hat{\boldsymbol{x}}_{k}$. This simplifies (10) to

$A(i)=\left.\frac{\partial f}{\partial x}\right|_{\begin{array}{l}u(i)=\hat{u}_{k} \\ x(i)=\hat{x}_{k}\end{array}}:=\hat{A}_{k}, B(i)=\left.\frac{\partial f}{\partial u}\right|_{\begin{array}{l}u(i)=\hat{u}_{k} \\ x(i)=\hat{x}_{k}\end{array}}:=\hat{B}_{k}$
$C(i)=\left.\frac{\partial h}{\partial x}\right|_{\begin{array}{l}u(i)=\hat{u}_{k} \\ x(i)=\hat{x}_{k}\end{array}}:=\hat{C}_{k}, D(i)=\left.\frac{\partial h}{\partial u}\right|_{\begin{array}{l}u(i)=\hat{u}_{k} \\ x(i)=\hat{x}_{k}\end{array}}:=\hat{D}_{k}$

and then (11) can be rewritten as $\nabla \boldsymbol{g}\left(\hat{\boldsymbol{u}}_{k}\right)=$

$$
\left[\begin{array}{ccccc}
\hat{D}_{k} & 0 & 0 & \cdots & 0 \\
\hat{C}_{k} \hat{B}_{k} & \hat{D}_{k} & 0 & \cdots & 0 \\
\hat{C}_{k} \hat{A}_{k} \hat{B}_{k} & \hat{C}_{k} \hat{B}_{k} & \hat{D}_{k} & \cdots & 0 \\
\vdots & \vdots & \vdots & \ddots & \vdots \\
\hat{C}_{k} \hat{A}_{k}^{N-2} \hat{B}_{k} & \hat{C}_{k} \hat{A}_{k}^{N-3} \hat{B}_{k} \hat{C}_{k} \hat{A}_{k}^{N-4} \hat{B}_{k} & \cdots & \hat{D}_{k}
\end{array}\right],
$$

where $\nabla \boldsymbol{g}\left(\hat{\boldsymbol{u}}_{k}\right) \in \mathbb{R}^{N \times N}$. The first-order Taylor expansion of $\boldsymbol{g}(\boldsymbol{u})$ around $\hat{\boldsymbol{u}}_{k}$ is used to produce $\nabla \boldsymbol{g}\left(\hat{\boldsymbol{u}}_{k}\right)^{\top} \boldsymbol{e}_{k}$ in (13) through the relation

$\boldsymbol{g}(\boldsymbol{u}) \approx \boldsymbol{g}\left(\hat{\boldsymbol{u}}_{k}\right)+\nabla \boldsymbol{g}\left(\hat{\boldsymbol{u}}_{k}\right)\left(\boldsymbol{u}-\hat{\boldsymbol{u}}_{k}\right)$.

Definition 1 A time reversal operator is now defined to flip the elements of a vector, i.e. the time reversal of $\boldsymbol{s}=[s(0), s(1), \ldots, s(N-1)]^{\top} \in \mathbb{R}^{N}$ is $\tilde{\boldsymbol{s}}=[s(N-$ $1), s(N-2), \ldots, s(0)]^{\top}$. For any vectors $s_{1}, s_{2} \in \mathbb{R}^{N}$ and any scalar $\alpha \in \mathbb{R}$, the time reversal operator satisfies

$\overbrace{s_{1}+s_{2}}=\tilde{s}_{1}+\tilde{s}_{2}$ and $\overparen{\alpha s_{1}}=\alpha \tilde{s}_{1}$.
Selecting $\boldsymbol{u}=\hat{\boldsymbol{u}}_{k}+\alpha \tilde{\boldsymbol{e}}_{k}$ with sufficiently small $\alpha>0$, gives rise to the approximation

$\boldsymbol{g}\left(\hat{\boldsymbol{u}}_{k}+\alpha \tilde{\boldsymbol{e}}_{k}\right) \approx \boldsymbol{g}\left(\hat{\boldsymbol{u}}_{k}\right)+\nabla \boldsymbol{g}\left(\hat{\boldsymbol{u}}_{k}\right)\left(\hat{\boldsymbol{u}}_{k}+\alpha \tilde{\boldsymbol{e}}_{k}-\hat{\boldsymbol{u}}_{k}\right)$

so that

$\nabla \boldsymbol{g}\left(\hat{\boldsymbol{u}}_{k}\right) \tilde{\boldsymbol{e}}_{k} \approx \frac{1}{\alpha}\left(\boldsymbol{g}\left(\hat{\boldsymbol{u}}_{k}+\alpha \tilde{\boldsymbol{e}}_{k}\right)-\boldsymbol{g}\left(\hat{\boldsymbol{u}}_{k}\right)\right)$.

Using the definition of the time reversal operator and the Taylor expansion of the system dynamics, the following proposition holds, with the proof given in Appendix A.

Proposition 1 Using symbols defined above, the time reversal of equation (18) is

$\overbrace{\nabla \boldsymbol{g}\left(\hat{\boldsymbol{u}}_{k}\right) \tilde{\boldsymbol{e}}_{k}}=\nabla \boldsymbol{g}\left(\hat{\boldsymbol{u}}_{k}\right)^{\top} \boldsymbol{e}_{k}$.

Then, the gradient ILC update law (13) can be rewritten as

$\boldsymbol{u}_{k+1}=\boldsymbol{u}_{k}+\frac{\beta}{\alpha}\left(\widetilde{\boldsymbol{g}\left(\hat{\boldsymbol{u}}_{k}+\alpha \tilde{\boldsymbol{e}}_{k}\right)}-\overparen{\boldsymbol{g}\left(\hat{\boldsymbol{u}}_{k}\right)}\right)$.

Remark 1 The attractive performance of gradient-based ILC approaches derives from utilizing model information within the update law. According to the above proposition, the model information can be substituted by the outputs of two extra experiments, the first of which involves applying the fixed operating point $\hat{\boldsymbol{u}}_{k}$ to the system and the second involves applying the sum of the operating point and the time reversal of the last tracking error. Thus, the gradient ILC update is realized in a data-driven manner.

The overall algorithm can be summarised as:

Algorithm 1 data-driven ILC for nonlinear systems

1. At trial $k, \boldsymbol{u}_{k}$ is applied to the system to generate $\boldsymbol{y}_{k}$

2. $\hat{\boldsymbol{u}}_{k}$ is calculated following equation (14) and is applied to the system to generate output $\boldsymbol{g}\left(\hat{\boldsymbol{u}}_{k}\right)$

3. Tracking error $\boldsymbol{e}_{k}=\boldsymbol{r}-\boldsymbol{y}_{k}$ is computed, $\hat{\boldsymbol{u}}_{k}+$ $\alpha \tilde{\boldsymbol{e}}_{k}$ is then applied to the system to generate the corresponding output is $\boldsymbol{g}\left(\hat{\boldsymbol{u}}_{k}+\alpha \tilde{\boldsymbol{e}}_{k}\right)$

4. The input for trial $k+1$ is updated using (20)

5. Increment $k$ and go to (1).

\section{Convergence Analysis}

In this section, the convergence conditions for the proposed data-driven gradient ILC approach are established. The main results are as follows.

For the system model (1) with the input update law (20), the following theorem holds. 
Theorem 1 Assuming the system dynamics $\boldsymbol{g}(\cdot)$ and the error norm $J(\cdot)$ are Lipschitz continuous gradient with constants $M, L$ respectively. The tracking error norm $J\left(\boldsymbol{u}_{k}\right)=\left\|\boldsymbol{e}_{k}\right\|^{2}$ monotonically converges, if the operating point deviation $\boldsymbol{u}_{d}=\hat{\boldsymbol{u}}_{k}-\boldsymbol{u}_{k}$ is bounded by

$\left\|\boldsymbol{u}_{d}\right\| \leq \frac{\left\|\nabla \boldsymbol{g}\left(\boldsymbol{u}_{k}\right)\right\|}{M}$

and the step length satisfies

$\beta<\frac{2}{L}\left(\frac{1-c}{1+2 c}\right)$

where $c=\frac{\left\|\boldsymbol{u}_{d}\right\| M}{\left\|\nabla \boldsymbol{g}\left(\boldsymbol{u}_{k}\right)\right\|}$.

Proof Expanding $J\left(\boldsymbol{u}_{k+1}\right)$ around $J\left(\boldsymbol{u}_{k}\right)$ gives

$$
\begin{aligned}
J\left(\boldsymbol{u}_{k+1}\right) \leq & J\left(\boldsymbol{u}_{k}\right)+\nabla J\left(\boldsymbol{u}_{k}\right)^{\top}\left(\boldsymbol{u}_{k+1}-\boldsymbol{u}_{k}\right) \\
& +\frac{1}{2} \nabla^{2} J\left(\boldsymbol{u}_{k}\right)\left\|\boldsymbol{u}_{k+1}-\boldsymbol{u}_{k}\right\|^{2}
\end{aligned}
$$

As $J(\cdot)$ is Lipschitz continuous gradient with constant $L$, we have $\nabla^{2} J(\boldsymbol{x}) \preceq L I$, and recalling the update law (13) of

$\boldsymbol{u}_{k+1}=\boldsymbol{u}_{k}+\beta\left(\nabla \boldsymbol{g}\left(\hat{\boldsymbol{u}}_{k}\right)\right)^{\top} \boldsymbol{e}_{k}$

Using the two equations, we get

$$
\begin{aligned}
J\left(\boldsymbol{u}_{k+1}\right) \leq & J\left(\boldsymbol{u}_{k}\right) \\
& +\nabla J\left(\boldsymbol{u}_{k}\right)^{\top}\left(\boldsymbol{u}_{k}+\beta\left(\nabla \boldsymbol{g}\left(\hat{\boldsymbol{u}}_{k}\right)\right)^{\top} \boldsymbol{e}_{k}-\boldsymbol{u}_{k}\right) \\
& +\frac{1}{2} L\left\|\boldsymbol{u}_{k}+\beta\left(\nabla \boldsymbol{g}\left(\hat{\boldsymbol{u}}_{k}\right)\right)^{\top} \boldsymbol{e}_{k}-\boldsymbol{u}_{k}\right\|^{2} \\
= & J\left(\boldsymbol{u}_{k}\right)+\beta \nabla J\left(\boldsymbol{u}_{k}\right)^{\top}\left(\nabla \boldsymbol{g}\left(\hat{\boldsymbol{u}}_{k}\right)\right)^{\top} \boldsymbol{e}_{k} \\
& +\frac{L \beta^{2}}{2}\left\|\left(\nabla \boldsymbol{g}\left(\hat{\boldsymbol{u}}_{k}\right)\right)^{\top} \boldsymbol{e}_{k}\right\|^{2} .
\end{aligned}
$$

Similarly for $\boldsymbol{J}(\cdot)$, we have $\nabla^{2} \boldsymbol{g}\left(\boldsymbol{u}_{k}\right) \preceq M I$. Then expanding $\nabla \boldsymbol{g}\left(\hat{\boldsymbol{u}}_{k}\right)$ about $\nabla \boldsymbol{g}\left(\boldsymbol{u}_{k}\right)$ gives

$$
\begin{aligned}
\nabla \boldsymbol{g}\left(\hat{\boldsymbol{u}}_{k}\right) & \leq \nabla \boldsymbol{g}\left(\boldsymbol{u}_{k}\right)+M\left\|\hat{\boldsymbol{u}}_{k}-\boldsymbol{u}_{k}\right\| \\
& =\nabla \boldsymbol{g}\left(\boldsymbol{u}_{k}\right)+M\left\|\boldsymbol{u}_{d}\right\|
\end{aligned}
$$

which yields

$$
\begin{aligned}
J\left(\boldsymbol{u}_{k+1}\right) \leq & J\left(\boldsymbol{u}_{k}\right)+\beta \nabla J\left(\boldsymbol{u}_{k}\right)^{\top}\left(\nabla \boldsymbol{g}\left(\boldsymbol{u}_{k}\right)\right)^{\top} \boldsymbol{e}_{k} \\
& +\frac{L \beta^{2}}{2}\left\|\left(\nabla \boldsymbol{g}\left(\boldsymbol{u}_{k}\right)\right)^{\top} \boldsymbol{e}_{k}\right\|^{2} \\
& +\beta \nabla J\left(\boldsymbol{u}_{k}\right)^{\top} M \boldsymbol{e}_{k}\left\|\boldsymbol{u}_{d}\right\| \\
& +\frac{L \beta^{2}}{2} \boldsymbol{e}_{k}^{\top} \nabla \boldsymbol{g}\left(\boldsymbol{u}_{k}\right) M \boldsymbol{e}_{k}\left\|\boldsymbol{u}_{d}\right\| \\
& +\frac{L \beta^{2}}{2} \boldsymbol{e}_{k}^{\top} M\left(\nabla \boldsymbol{g}\left(\boldsymbol{u}_{k}\right)\right)^{\top} \boldsymbol{e}_{k}\left\|\boldsymbol{u}_{d}\right\| \\
= & J\left(\boldsymbol{u}_{k}\right)-\beta\left(\left\|\nabla J\left(\boldsymbol{u}_{k}\right)\right\|^{2}\right. \\
& \left.-\left\|\nabla J\left(\boldsymbol{u}_{k}\right)\right\|\left\|\boldsymbol{e}_{k}\right\| M\left\|\boldsymbol{u}_{d}\right\|\right) \\
& +\beta^{2}\left(\frac{1}{2} L\left\|\nabla J\left(\boldsymbol{u}_{k}\right)\right\|^{2}\right. \\
& \left.+L\left\|\nabla J\left(\boldsymbol{u}_{k}\right)\right\|\left\|\boldsymbol{e}_{k}\right\| M\left\|\boldsymbol{u}_{d}\right\|\right) .
\end{aligned}
$$

To ensure monotonic convergence of the tracking error norm, that is $J\left(\boldsymbol{u}_{k+1}\right)<J\left(\boldsymbol{u}_{k}\right)$ for any $k \in \mathbb{N}^{+}$, we first require

$0<\left\|\nabla J\left(\boldsymbol{u}_{k}\right)\right\|^{2}-\left\|\nabla J\left(\boldsymbol{u}_{k}\right)\right\|\left\|\boldsymbol{e}_{k}\right\| M\left\|\boldsymbol{u}_{d}\right\|$

which is guaranteed by (21), and then we require

$$
\begin{aligned}
& \beta^{2} L\left(\frac{1}{2}\left\|\nabla J\left(\boldsymbol{u}_{k}\right)\right\|^{2}+\left\|\nabla J\left(\boldsymbol{u}_{k}\right)\right\|\left\|\boldsymbol{e}_{k}\right\| M\left\|\boldsymbol{u}_{d}\right\|\right) \\
& <\beta\left(\left\|\nabla J\left(\boldsymbol{u}_{k}\right)\right\|^{2}-\left\|\nabla J\left(\boldsymbol{u}_{k}\right)\right\|\left\|\boldsymbol{e}_{k}\right\| M\left\|\boldsymbol{u}_{d}\right\|\right)
\end{aligned}
$$

so that

$\beta<\frac{2}{L} \frac{\left\|\nabla J\left(\boldsymbol{u}_{k}\right)\right\|^{2}-\left\|\nabla J\left(\boldsymbol{u}_{k}\right)\right\|\left\|\boldsymbol{e}_{k}\right\| M\left\|\boldsymbol{u}_{d}\right\|}{\left\|\nabla J\left(\boldsymbol{u}_{k}\right)\right\|^{2}+2\left\|\nabla J\left(\boldsymbol{u}_{k}\right)\right\|\left\|\boldsymbol{e}_{k}\right\| M\left\|\boldsymbol{u}_{d}\right\|}$

which yields (22)

Remark 2 Satisfying (21) is not always possible since it contains no directly tuneable parameters. However, the designer can seek to satisfy it indirectly in two ways:

- In many applications there is freedom available in the choice of reference trajectory, for example employing smoother or slower choices can always enable it to be satisfied. This approach is not dissimilar to the standard use of 'learning filters' in ILC that prevent learning at higher frequencies, see e.g. [8].

- Another route is to maintain the reference, but instead add a feedback controller around the plant. Often called a pre-stablizing controller, this is also common practice in ILC to improve baseline disturbance rejection and initial tracking [8]. In our case it can be used to modify $\boldsymbol{g}(\cdot)$ and $M$ and so enable (21) to be satisfied. 
System nonlinearities have the effect of reducing the value of $\beta$ in (22) due to the truncation employed in inequalities (23), (24). Note that $\beta$ and $c$ are computed every trial to reduce conservatism (which scales with the size of higher order terms in the Taylor expansion). However, to reduce computational effort (22) can be replaced using a fixed value of $c$ given by

$c=\max _{k \in \mathbb{N}} \frac{\left\|\boldsymbol{u}_{d}\right\| M}{\left\|\nabla \boldsymbol{g}\left(\boldsymbol{u}_{k}\right)\right\|}$

Alternatively, $\beta$ may be chosen heuristically and fixed for all trials. When applying the update law (20) to linear systems, Theorem 1 can be specialised to the following lemma.

Lemma 1 If system $\boldsymbol{g}(\cdot)$ is linear and $\nabla \boldsymbol{g}\left(\boldsymbol{u}_{k}\right)=G \forall k \in$ $\mathbb{N}^{+}$where $G \in \mathbb{R}^{N \times N}$ is constant, then Theorem 1 holds with $M=0$ and $L=\|G\|^{2}$. Step length bound (22) therefore becomes

$\beta<\frac{2}{\|G\|^{2}}$

and coincides with the convergence condition of modelbased gradient ILC for linear systems in [34].

Proof Note that $L$ captures the system nonlinearity as $\nabla^{2} J\left(\boldsymbol{u}_{k}\right)=\nabla\left(-\nabla \boldsymbol{g}\left(\boldsymbol{u}_{k}\right) \boldsymbol{e}_{k}\right)$

$$
=-\left(\nabla^{2} \boldsymbol{g}\left(\boldsymbol{u}_{k}\right)\right) \boldsymbol{e}_{k}-\nabla \boldsymbol{g}\left(\boldsymbol{u}_{k}\right)\left(\nabla g\left(\boldsymbol{u}_{k}\right)^{\top}\right)
$$

so if the system is linear, we have $\nabla^{2} \boldsymbol{g}\left(\boldsymbol{u}_{k}\right)=0$, and then

$\nabla^{2} J\left(\boldsymbol{u}_{k}\right)=-\nabla \boldsymbol{g}\left(\boldsymbol{u}_{k}\right)\left(\nabla \boldsymbol{g}\left(\boldsymbol{u}_{k}\right)^{\top}\right)$

so $L$ can be selected as $\left\|\nabla \boldsymbol{g}\left(\boldsymbol{u}_{k}\right)\left(\nabla \boldsymbol{g}\left(\boldsymbol{u}_{k}\right)^{\top}\right)\right\|$.

The next lemma shows that the Lipschitz condition of $J(\cdot)$ could be replaced by a more conservative value which only relates to the system dynamics $\boldsymbol{g}(\cdot)$.

Lemma 2 Theorem 1 holds if the Lipschitz constant $L$ replaced by

$\max _{\boldsymbol{u} \in \mathbb{R}^{N}}\left\{\|\nabla \boldsymbol{g}(\boldsymbol{u})\|^{2}\right\}+M\left\|\boldsymbol{e}_{0}\right\|$.

Proof Follows from equation (28) and noting that $\left\|\boldsymbol{e}_{0}\right\|$ is the maximum value of $\left\|\boldsymbol{e}_{k}\right\|$.

If $\left\|\hat{\boldsymbol{u}}_{k}-\boldsymbol{u}_{k}\right\|=0$ can be guaranteed for all trials through the selection of fixed operating points, or if the system is linear, that is $M=0$, then Theorem 1 holds with step length bound (22) replaced by

$\beta<\frac{2}{L}$.

For a specific choice of step length $\beta$ satisfying (26), the monotonic tracking error bound is given by the following theorem.
Theorem 2 Under the conditions of Theorem 1, if the step length $\beta$ in update (20) is selected as

$\beta=\frac{1}{L}\left(\frac{1-c}{1+2 c}\right)$.

then the tracking error norm is bounded monotonically as

$\left\|\boldsymbol{e}_{k+1}\right\|^{2} \leq \prod_{i=0}^{k}\left(1-\frac{\beta}{2}(1-c) \underline{\sigma}\left(\nabla \boldsymbol{g}\left(\boldsymbol{u}_{i}\right)\left(\nabla \boldsymbol{g}\left(\boldsymbol{u}_{i}\right)\right)^{\top}\right)\right)\left\|\boldsymbol{e}_{0}\right\|^{2}$

and

$\frac{1}{2} \leq\left(1-\frac{\beta}{2}(1-c) \underline{\sigma}\left(\nabla \boldsymbol{g}\left(\boldsymbol{u}_{i}\right)\left(\nabla \boldsymbol{g}\left(\boldsymbol{u}_{i}\right)\right)^{\top}\right)\right)<1$.

Proof First recall (25)

$$
\begin{aligned}
J\left(\boldsymbol{u}_{k+1}\right) \leq & J\left(\boldsymbol{u}_{k}\right)-\beta\left(\left\|\nabla J\left(\boldsymbol{u}_{k}\right)\right\|^{2}\right. \\
& \left.-\left\|\nabla J\left(\boldsymbol{u}_{k}\right)\right\|\left\|\boldsymbol{e}_{k}\right\| M\left\|\hat{\boldsymbol{u}}_{k}-\boldsymbol{u}_{k}\right\|\right) \\
& +\beta^{2} L\left(\frac{1}{2}\left\|\nabla J\left(\boldsymbol{u}_{k}\right)\right\|^{2}\right. \\
& \left.+\|\nabla J(x)\| \boldsymbol{e}_{k}\|M\| \hat{\boldsymbol{u}}_{k}-\boldsymbol{u}_{k} \|\right) \\
= & J\left(\boldsymbol{u}_{k}\right)-\beta\left(\left\|\nabla J\left(\boldsymbol{u}_{k}\right)\right\|^{2}-c\left\|\nabla J\left(\boldsymbol{u}_{k}\right)\right\|^{2}\right) \\
& \quad+\beta^{2} L\left(\frac{1}{2}\left\|\nabla J\left(\boldsymbol{u}_{k}\right)\right\|^{2}+c\left\|\nabla J\left(\boldsymbol{u}_{k}\right)\right\|^{2}\right) \\
= & J\left(\boldsymbol{u}_{k}\right)-\beta(1-c)\left\|\nabla J\left(\boldsymbol{u}_{k}\right)\right\|^{2} \\
& +\beta^{2} L\left(\frac{1}{2}+c\right)\left\|\nabla J\left(\boldsymbol{u}_{k}\right)\right\|^{2} .
\end{aligned}
$$

Therefore it is necessary that

$\beta^{2} L\left(\frac{1}{2}+c\right)\left\|\nabla J\left(\boldsymbol{u}_{k}\right)\right\|^{2}<\beta(1-c)\left\|\nabla J\left(\boldsymbol{u}_{k}\right)\right\|^{2}$

which corresponds to

$\beta<\frac{2}{L}\left(\frac{1-c}{1+2 c}\right)$.

Selecting half this maximum value, i.e. $\beta$ is given by (32), means that (35) becomes

$$
\begin{aligned}
J\left(\boldsymbol{u}_{k+1}\right) \leq & J\left(\boldsymbol{u}_{k}\right)-\beta(1-c)\left\|\nabla J\left(\boldsymbol{u}_{k}\right)\right\|^{2} \\
& +\beta^{2} L\left(\frac{1}{2}+c\right)\left\|\nabla J\left(\boldsymbol{u}_{k}\right)\right\|^{2} \\
= & J\left(\boldsymbol{u}_{k}\right)-\beta(1-c)\left\|\nabla J\left(\boldsymbol{u}_{k}\right)\right\|^{2} \\
& +2 \beta\left(\frac{1-c}{1+2 c}\right)\left(\frac{1}{2}+c\right)\left\|\nabla J\left(\boldsymbol{u}_{k}\right)\right\|^{2} \\
= & J\left(\boldsymbol{u}_{k}\right)-\beta(1-c)\left\|\nabla J\left(\boldsymbol{u}_{k}\right)\right\|^{2} \\
& +\beta\left(\frac{1-c}{2}\right)\left\|\nabla J\left(\boldsymbol{u}_{k}\right)\right\|^{2} \\
= & J\left(\boldsymbol{u}_{k}\right)-\frac{\beta}{2}(1-c)\left\|\nabla J\left(\boldsymbol{u}_{k}\right)\right\|^{2} .
\end{aligned}
$$


Writing

$$
\begin{aligned}
\left\|\nabla J\left(\boldsymbol{u}_{k}\right)\right\|^{2} & =\left\|\left(\nabla \boldsymbol{g}\left(\hat{\boldsymbol{u}}_{k}\right)\right)^{\top} \boldsymbol{e}_{k}\right\|^{2} \\
& \geq \underline{\sigma}\left(\nabla \boldsymbol{g}\left(\boldsymbol{u}_{k}\right)\left(\nabla \boldsymbol{g}\left(\boldsymbol{u}_{k}\right)\right)^{\top}\right)\left\|\boldsymbol{e}_{k}\right\|^{2}
\end{aligned}
$$

so that (37) becomes

$$
\begin{aligned}
J\left(\boldsymbol{u}_{k+1}\right) \leq & J\left(\boldsymbol{u}_{k}\right)- \\
& \frac{\beta}{2}(1-c) \underline{\sigma}\left(\nabla \boldsymbol{g}\left(\boldsymbol{u}_{k}\right)\left(\nabla \boldsymbol{g}\left(\boldsymbol{u}_{k}\right)\right)^{\top}\right)\left\|\boldsymbol{e}_{k}\right\|^{2} .
\end{aligned}
$$

Thus, we have

$$
\begin{gathered}
\left\|\boldsymbol{e}_{k+1}\right\|^{2} \leq\left(1-\frac{\beta}{2}(1-c) \underline{\sigma}\left(\nabla \boldsymbol{g}\left(\boldsymbol{u}_{k}\right)\left(\nabla \boldsymbol{g}\left(\boldsymbol{u}_{k}\right)\right)^{\top}\right)\right)\left\|\boldsymbol{e}_{k}\right\|^{2} \\
\leq \prod_{i=0}^{k}\left(1-\frac{\beta}{2}(1-c) \underline{\sigma}\left(\nabla \boldsymbol{g}\left(\boldsymbol{u}_{i}\right)\left(\nabla \boldsymbol{g}\left(\boldsymbol{u}_{i}\right)\right)^{\top}\right)\right)\left\|\boldsymbol{e}_{0}\right\|^{2} .
\end{gathered}
$$

Relation (34) follows from (32), and since $0 \leq c<1$ and $0<\underline{\sigma}\left(\nabla \boldsymbol{g}\left(\boldsymbol{u}_{i}\right)\left(\nabla \boldsymbol{g}\left(\boldsymbol{u}_{i}\right)\right)^{\top}\right) \leq L$.

As noted, system nonlinearities have the effect of reducing the maximum value of $\beta$ that can be used. This in turn reduces the convergence rate since each term in the product within (33) becomes closer to unity.

Lemma 3 If system $\boldsymbol{g}(\cdot)$ is linear (i.e. $\nabla \boldsymbol{g}\left(\boldsymbol{u}_{k}\right)=G \forall k$ ) then Theorem 2 holds with $c=0$ and $\nabla \boldsymbol{g}\left(\boldsymbol{u}_{i}\right)=G$. In particular the error is monotonically bounded as

$$
\left(1-\frac{\beta}{2} \bar{\sigma}\left(G G^{\top}\right)\right)^{k} \leq \frac{\left\|\boldsymbol{e}_{k}\right\|^{2}}{\left\|\boldsymbol{e}_{0}\right\|^{2}} \leq\left(1-\frac{\beta}{2} \underline{\sigma}\left(G G^{\top}\right)\right)^{k}
$$

This coincides with the bounds derived in [15] for modelbased gradient ILC.

\section{Data-driven Gradient-based Point-to-point ILC}

A limitation of data-driven gradient ILC is that it may converge slowly. To address this, suppose that the plant output is only required to track a reference trajectory at a fixed number, $M \leq N$, of sample instants along the trial duration. Denote these sample instants by $0 \leq$ $n_{1}<n_{2}<\cdots<n_{M}<N$ and the corresponding 'pointto-point' reference locations as $r_{1}, r_{2}, \cdots, r_{M}$. To define the point-to-point tracking problem it is first necessary to remove the points that do not need to be tracked from the original reference or output. This is achieved by defining matrix $\Phi \in \mathbb{R}^{M \times N}$ with elements

$\Phi_{i, j}=\left\{\begin{array}{cc}1 & \text { if } j=n_{i} \\ 0 & \text { otherwise }\end{array}\right.$.

When any output vector is pre-multiplied by $\Phi$, it extracts the components that correspond to prescribed point-to-point locations, while retaining the order in which they appear. For example, $\Phi \boldsymbol{r}=\left[r_{1}, r_{2}, \cdots, r_{M}\right]^{\top}$ $\in \mathbb{R}^{M}$. For simplicity, denote the point-to-point reference by $\boldsymbol{r}^{\Phi}:=\Phi \boldsymbol{r}$ and the point-to-point outputs as $\boldsymbol{y}^{\Phi}:=\Phi \boldsymbol{y}$. We then modify the task (6) as

$J^{\Phi}\left(\boldsymbol{u}_{k}\right):=\left\|\boldsymbol{e}_{k}^{\Phi}\right\|^{2}, \quad \boldsymbol{e}_{k}^{\Phi}=\boldsymbol{r}^{\Phi}-\Phi \boldsymbol{g}\left(\boldsymbol{u}_{k}\right)$

and the ILC problem (5) becomes

$\lim _{k \rightarrow \infty} \boldsymbol{u}_{k}=\boldsymbol{u}_{\infty}, \quad \lim _{k \rightarrow \infty} \boldsymbol{e}_{k}^{\Phi}=\mathbf{0}$.

Let the update law (7) be replaced by

$$
\begin{aligned}
\boldsymbol{u}_{k+1} & =\boldsymbol{u}_{k}+\beta\left(\Phi \nabla \boldsymbol{g}\left(\boldsymbol{u}_{k}\right)\right)^{\top} \Phi \boldsymbol{e}_{k} \\
& =\boldsymbol{u}_{k}+\beta \nabla \boldsymbol{g}\left(\boldsymbol{u}_{k}\right)^{\top} \Phi^{\top} \Phi \boldsymbol{e}_{k}
\end{aligned}
$$

then it can be shown that relation (59) becomes

$\nabla \boldsymbol{g}\left(\hat{\boldsymbol{u}}_{k}\right)^{\top} \Phi^{\top} \Phi \boldsymbol{e}_{k}=\frac{1}{\alpha}\left(\widetilde{\boldsymbol{g}\left(\hat{\boldsymbol{u}}_{k}+\alpha \overparen{\left.\Phi^{\top} \Phi \boldsymbol{e}_{k}\right)}\right.}-\overparen{\boldsymbol{g}\left(\hat{\boldsymbol{u}}_{k}\right)}\right)$

so Algorithm 1 changes to

Algorithm 2 point-to-point data-driven ILC

1. At trial $k, \boldsymbol{u}_{k}$ is applied to the system to generate $\boldsymbol{y}_{k}$

2. $\hat{\boldsymbol{u}}_{k}$ is calculated using equation (14) and is applied to the system to generate output $\boldsymbol{g}\left(\hat{\boldsymbol{u}}_{k}\right)$

3. Tracking error $\Phi^{\top} \boldsymbol{e}_{k}^{\Phi}=\Phi^{\top}\left(\boldsymbol{r}-\boldsymbol{y}_{k}\right)$ is computed, $\hat{\boldsymbol{u}}_{k}+\alpha \overparen{\Phi^{\top} \boldsymbol{e}_{k}^{\Phi}}$ is applied to the system and the corresponding output is $\boldsymbol{g}\left(\hat{\boldsymbol{u}}_{k}+\alpha \overparen{\Phi^{\top} \boldsymbol{e}_{k}^{\Phi}}\right)$

4. the input for trial $k+1$ is updated using (44) and (45), i.e.

$$
\boldsymbol{u}_{k+1}=\boldsymbol{u}_{k}+\frac{\beta}{\alpha}(\overbrace{\boldsymbol{g}\left(\hat{\boldsymbol{u}}_{k}+\alpha \overparen{\Phi^{\top} \boldsymbol{e}_{k}^{\Phi}}\right)}-\overparen{\boldsymbol{g}\left(\hat{\boldsymbol{u}}_{k}\right)})
$$

5. Increment $k$ and go to (1).

Our previous results are hence modified as:

Theorem 3 Assuming the system dynamics $\boldsymbol{g}(\cdot)$ and the error norm $J^{\Phi}(\cdot)$ are Lipschitz continuous gradient with constants $M, L^{\Phi}$ respectively. The error norm $J^{\Phi}\left(\boldsymbol{u}_{k}\right)=\left\|\boldsymbol{e}_{k}^{\Phi}\right\|^{2}$ monotonically converges, if the operating point deviation $\boldsymbol{u}_{d}=\hat{\boldsymbol{u}}_{k}-\boldsymbol{u}_{k}$ is bounded by

$\left\|\boldsymbol{u}_{d}\right\| \leq \frac{\left\|\nabla \boldsymbol{g}\left(\boldsymbol{u}_{k}\right)\right\|}{M}$

and the step length satisfies

$\beta<\frac{2}{L^{\Phi}}\left(\frac{1-c}{1+2 c}\right)$

where $c=\frac{\left\|\boldsymbol{u}_{d}\right\| M}{\left\|\nabla \boldsymbol{g}\left(\boldsymbol{u}_{k}\right)\right\|}$. 
Note (47) is the same as condition (21) in Theorem 1 . However $\beta$ will be significantly larger due to the smaller value of $L^{\Phi}$, as illustrated next.

Lemma 4 If system $\boldsymbol{g}(\cdot)$ is linear and $\nabla \boldsymbol{g}\left(\boldsymbol{u}_{k}\right)=G \forall k \in$ $\mathbb{N}^{+}$where $G \in \mathbb{R}^{N \times N}$ is constant, then Theorem 3 holds with $M=0$ and $L^{\Phi}=\|\Phi G\|^{2}$. In particular $L^{\Phi}$ decreases as each point-to-point location is removed.

Proof In the linear case

$L^{\Phi}=\left\|\Phi \nabla \boldsymbol{g}\left(\boldsymbol{u}_{k}\right)\left(\nabla \boldsymbol{g}\left(\boldsymbol{u}_{k}\right)^{\top}\right) \Phi^{\top}\right\|=\bar{\sigma}\left(\Phi G(\Phi G)^{\top}\right)$.

Let the $M$ eigenvalues of the matrix $A=(\Phi G)(\Phi G)^{\top}$ be denoted $\lambda_{M} \leq \lambda_{M-1} \cdots \leq \lambda_{2} \leq \lambda_{1}$, which also equal the singular values since $A$ is Normal. Suppose $\tilde{\Phi}$ corresponds to $\Phi$ but with one row (i.e, point-to-point location) removed. Let the $M-1$ eigenvalues of the matrix $B=(\tilde{\Phi} G)(\tilde{\Phi} G)^{\top}$ be denoted $\mu_{M} \leq \mu_{M-1} \cdots \leq$ $\mu_{3} \leq \mu_{2}$, which also equal the singular values since $B$ is Normal. Then $\lambda_{M} \leq \mu_{M} \cdots \leq \mu_{3} \leq \lambda_{2} \leq \mu_{2} \leq \lambda_{1}$ by Cauchy's interlace theorem. If the eigenvalues of $B$ are distinct and no eigenvector of $B$ is orthogonal to the $i^{\text {th }}$ column of $A$ with the $i^{\text {th }}$ element removed, then $\lambda_{M}<\mu_{M} \cdots<\mu_{3}<\lambda_{2}<\mu_{2}<\lambda_{1}$. It follows that

$$
\begin{aligned}
\underline{\sigma}\left(\Phi G(\Phi G)^{\top}\right)<\underline{\sigma}\left(\tilde{\Phi} G(\tilde{\Phi} G)^{\top}\right)< \\
\bar{\sigma}\left(\tilde{\Phi} G(\tilde{\Phi} G)^{\top}\right)<\bar{\sigma}\left(\Phi G(\Phi G)^{\top}\right)
\end{aligned}
$$

Theorem 4 Under the conditions of Theorem 3, the tracking error norm is bounded monotonically as

$$
\begin{aligned}
\left\|\boldsymbol{e}_{k+1}^{\Phi}\right\|^{2} \leq \prod_{i=0}^{k}\left(1-\frac{\beta}{2}(1-c) \underline{\sigma}\left(\Phi \nabla \boldsymbol{g}\left(\boldsymbol{u}_{i}\right)(\Phi\right.\right. & \left.\left.\left.\nabla \boldsymbol{g}\left(\boldsymbol{u}_{i}\right)\right)^{\top}\right)\right) \\
& \times\left\|\boldsymbol{e}_{0}^{\Phi}\right\|^{2}
\end{aligned}
$$

if the step length $\beta$ in update (46) is selected as

$\beta=\frac{1}{L^{\Phi}}\left(\frac{1-c}{1+2 c}\right)$

The convergence rate increases as each point-to-point location is removed.

Proof From relations (49) we have

$$
\begin{aligned}
(1- & \left.\frac{\beta}{2}(1-c) \underline{\sigma}\left(\Phi \nabla \boldsymbol{g}\left(\boldsymbol{u}_{i}\right)\left(\Phi \nabla \boldsymbol{g}\left(\boldsymbol{u}_{i}\right)\right)^{\top}\right)\right) \\
& <\left(1-\frac{\beta}{2}(1-c) \underline{\sigma}\left(\tilde{\Phi} \nabla \boldsymbol{g}\left(\boldsymbol{u}_{i}\right)\left(\tilde{\Phi} \nabla \boldsymbol{g}\left(\boldsymbol{u}_{i}\right)\right)^{\top}\right)\right)
\end{aligned}
$$

Lemma 5 If system $\boldsymbol{g}(\cdot)$ is linear (i.e. $\nabla \boldsymbol{g}\left(\boldsymbol{u}_{k}\right)=G \forall k$ ) then Theorem 4 holds with $c=0$ and $\nabla \boldsymbol{g}\left(\boldsymbol{u}_{i}\right)=G$. In particular the error is monotonically bounded as

$\left(1-\frac{\beta}{2} \bar{\sigma}\left(\Phi G(\Phi G)^{\top}\right)\right)^{k} \leq \frac{\left\|\boldsymbol{e}_{k}^{\Phi}\right\|^{2}}{\left\|\boldsymbol{e}_{0}^{\Phi}\right\|^{2}} \leq\left(1-\frac{\beta}{2} \underline{\sigma}\left(\Phi G(\Phi G)^{\top}\right)\right)^{k}$.
Moreover, if initial input $\boldsymbol{u}_{0}=0$, the input will converge to the minimum input solution that satisfies the pointto-point tracking task, i.e.

$\lim _{k \rightarrow \infty} \boldsymbol{u}_{k}=\boldsymbol{u}_{\infty}$

$\boldsymbol{u}_{\infty}:=\min _{\boldsymbol{u} \in \mathbb{R}^{N}}\|\boldsymbol{u}\|^{2}$ such that $\boldsymbol{r}^{\Phi}=\Phi G \boldsymbol{u}$.

Proof Note $\left(1-\frac{\beta}{2} \underline{\sigma}\left(\Phi G(\Phi G)^{\top}\right)\right)$ reduces as points are removed. $\left(1-\frac{\beta}{2} \bar{\sigma}\left(\Phi G(\Phi G)^{\top}\right)\right)$ increases as points are removed. Repeated application of the input update (44) yields the relation

$\boldsymbol{u}_{k+1}=\sum_{j=0}^{k}\left(I-\beta(\Phi G)^{\top} \Phi G\right)^{j} \beta(\Phi G)^{\top} \boldsymbol{r}^{\Phi}$

so that

$$
\begin{aligned}
\boldsymbol{u}_{\infty} & =\sum_{j=0}^{\infty}\left(I-\beta(\Phi G)^{\top} \Phi G\right)^{j} \beta(\Phi G)^{\top} \boldsymbol{r}^{\Phi} \\
& =\left(\beta(\Phi G)^{\top} \Phi G\right)^{-1} \beta(\Phi G)^{\top} \boldsymbol{r}^{\Phi} \\
& =\left((\Phi G)^{\top} \Phi G\right)^{-1}(\Phi G)^{\top} \boldsymbol{r}^{\Phi}
\end{aligned}
$$

which is the solution to (53).

\section{Experimental Application to Rehabilitation}

Every year fifteen million people suffer a stroke worldwide and only $5 \%$ of survivors with severe paralysis regain upper limb function [4]. Functional electrical stimulation (FES) is a well-established intervention for motor rehabilitation and involves artificially stimulating muscles to enable patients to practice functional task training. In recent clinical reviews FES has shown significant benefit in improving upper-limb performance of activities of daily living [16].

Effective rehabilitation requires that FES coincides with voluntary intention and therefore must accurately support the intended movement [37]. Commercial FES systems use open loop or triggered stimulation, and therefore do not provide effective therapy. ILC is one of the few control schemes that has been clinically applied to stroke participants, and has led to statistically significant improvements in outcome measures in several clinical trials with both stroke $[21,28,27]$ and multiple sclerosis participants [38]. However, the need for a dynamic model of the system meant that identification tests were required at the beginning of each treatment session, reducing the time available for therapy and increasing fatigue. This is a primary factor severely limiting more widespread use of FES in rehabilitation. 
The data-driven approach developed in this paper therefore has substantial potential to solve this problem. It will be applied to the specific problem of elbow flexion/extension, since this is a fundamental component of upper limb rehabilitation and is critical to functional recovery of patients.

\subsection{Hardware System}

The FES-based upper-limb rehabilitation system is shown in Fig. 1. Elbow extension/flexion is performed in the horizontal plane, matching previous clinical applications [21], and the elbow angle $y$ is measured by a electrogoniometer (Biometric Ltd., UK). Stimulation is produced using a commercial system (Odstock Medical Limited, UK) and FES pulsewidth is the controlled variable. Biceps and triceps muscles are stimulated synergistically (with respective pulsewidths $u_{B I}, u_{T R}$ ) using the co-activation function

$\left(u_{T R}, u_{B I}\right)=h_{C}(u):$

$u_{T R}=\left\{\begin{array}{r}-u, u \leq 0 \\ 0, u>0\end{array}, \quad u_{B I}=\left\{\begin{array}{l}u, u>0 \\ 0, u \leq 0\end{array}\right.\right.$.

The dynamics of each stimulated muscle comprise a Hammerstein structure consisting of a nonlinear isometric recruitment curve (IRC), $h_{I R C}(u)$, and linear activation dynamics (LAD), $H_{L A D}(s)$. The output of the Hammerstein blocks are multiplied by two nonlinear functions $g_{T R}(y, \dot{y}), g_{B I}(y, \dot{y})$ to capture the position and velocity dependence of stimulated muscle. The resulting models are nonlinear and vary rapidly due to effects of fatigue and spasticity [20]. The generated torque feeds into the forearm rigid body dynamics which comprise forearm inertia and a nonlinear damping and friction, $g_{a}(y, \dot{y})$. The resulting system structure

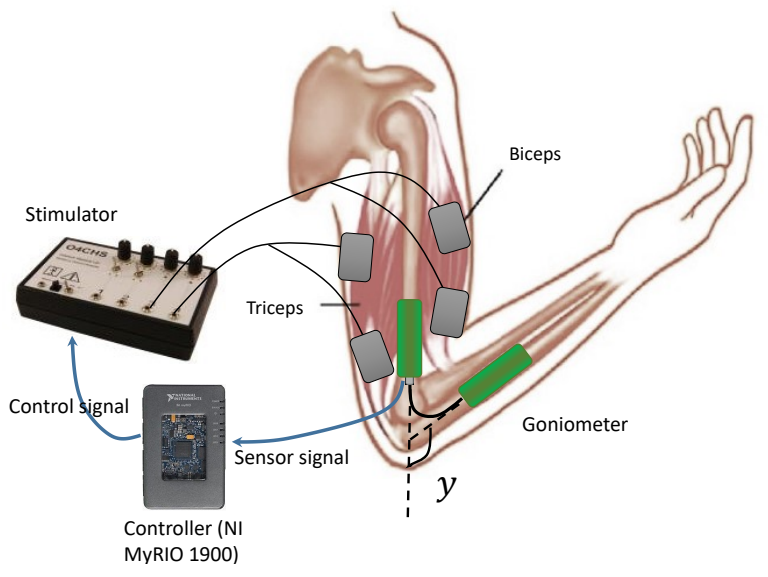

Fig. 1 FES-based elbow rehabilitation system.

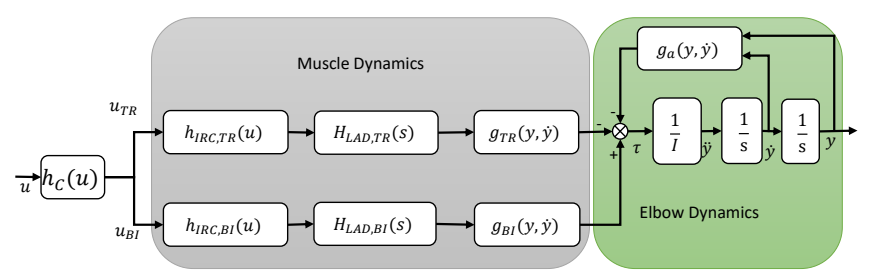

Fig. 2 FES assisted elbow dynamics.

is shown in Fig. 2, with subscripts denoting triceps and biceps components.

\subsection{Data-driven Algorithms}

The proposed data-driven ILC algorithms are compared with the existing linear data-driven ILC approach of $[10,6]$. This approach, termed Algorithm 3, is equivalent to setting $\hat{\boldsymbol{u}}_{k}=\mathbf{0}$ within Algorithm 1. It is important to note that this update guarantees convergence to zero error not only for linear systems, but also for classes of nonlinear systems with sufficiently mild non-linearities. It is therefore the most direct choice of approach for comparison, and indeed no other data-driven gradientbased ILC algorithms exist for nonlinear systems. The ILC methods used can therefore be summarised as

Algorithm 1 : data-driven gradient ILC approach given by Algorithm 1,

Algorithm 2 : data-driven point-to-point gradient ILC approach given by Algorithm 2,

Algorithm 3 : existing data-driven gradient ILC for linear systems from [10], which is a special case of Algorithm 1 and is described by:

Algorithm 3 linear data-driven ILC

1. At trial $k, \boldsymbol{u}_{k}$ is applied to the system to generate $\boldsymbol{y}_{k}$

2. Tracking error $\boldsymbol{e}_{k}=\boldsymbol{r}-\boldsymbol{y}_{k}$ is computed and timereversed to produce $\tilde{\boldsymbol{e}}_{k}$, then $\tilde{\boldsymbol{e}}_{k}$ is applied to the system and the corresponding output is measured and denoted $\boldsymbol{s}_{k}$

3. The input for trial $k+1$ is updated according to

$$
\boldsymbol{u}_{k+1}=\boldsymbol{u}_{k}+\beta \tilde{\boldsymbol{s}}_{k}
$$

4. Increment $k$ and go to (1).

\subsection{Reference Trajectories}

The reference trajectory $\boldsymbol{r}$ corresponds to smooth functional elbow extension/flexion with duration $10 \mathrm{~s}$, moving the elbow from 0 and to 40 degrees and then returning to 0 . This is similar to movements employed 
clinically in [21]. The sample frequency is $20 \mathrm{~Hz}$ so that $N=200$. The tracking error used by Algorithms 1 and 3 is $\boldsymbol{e}=\boldsymbol{r}-\boldsymbol{y}$, where $\boldsymbol{y}$ is the elbow angle defined in Fig. 1. The data-driven point-to-point ILC algorithm instead selects sample indices $n_{1}=0, n_{2}=40$, $n_{3}=100, n_{4}=160$ and $n_{5}=200$. The corresponding reference points are $r_{1}=0, r_{2}=0, r_{3}=40, n_{4}=0$ and $n_{5}=0$. The point-to-point error is measured only at the $M=5$ sample times and is denoted $\boldsymbol{e}^{\Phi}=\Phi(\boldsymbol{r}-\boldsymbol{y})$.

\subsection{Experimental Results and Discussion}

Five healthy participants (P1 to P5) were recruited to the study, following Ethics protocol ERGO/FEPS/47701 (more information about the Ethics protocol is given in the 'Research involving Human Participants' section). Each attended a single session of two hour duration.

In each session 20 trials were conducted for each of the three Algorithms. A gain of $\beta=0.2$ was used in Algorithms 1 and 3, and a gain of $\beta=8$ was used in Algorithm 2. A value of $\alpha=1$ was used throughout: in the analysis of Section 3 it was assumed that this perturbation term was negligibly small, however in practice a larger value is required to compensate for the presence of external disturbances and system dynamics that are not continuously differentiable.

A 20 minute rest period was provided between algorithms to ameliorate the effect of fatigue. For every trial, the trajectory tracking error norm $\left\|e_{k}\right\|$ is calculated, representing the tracking performance. For each session, the mean and minimum of error norms are computed and shown in Table 1, representing the performance of the algorithm and the best result respectively. Compared with Algorithm 3, the means and minimums $\left\|\boldsymbol{e}_{k}\right\|$ of Algorithm 1 are reduced by more than a half for the five participants, indicating that Algorithm 1 produces far superior results compared to Algorithm 3. The results of Algorithm 2 are partitioned in the table to reflect the fact that its tracking error, $\left\|\boldsymbol{e}_{k}^{\Phi}\right\|$, comprises only 5 points.

The data in Table 1 vary for different participants due to their diverse musculoskeletal characteristics and the presence of external disturbances (including measurement error, FES signal interference, and involuntary muscle activation unconsciously applied by the participant). Superior performance could be obtained by tuning the learning gain $\beta$ for each participant, however a uniform value has been selected to aid comparison between participants.

The error norm convergence for the five participants similarly varies, but all show a similar tendency. To illustrate the convergence process clearly, representative
Table 1 Mean and minimum of 2-norm of trajectory tracking error

\begin{tabular}{l|l|lllll}
\hline \multicolumn{2}{c|}{} & \multicolumn{5}{c}{ Participant } \\
\multicolumn{2}{c|}{} & \multicolumn{1}{c}{ P1 } & \multicolumn{1}{c}{ P2 } & \multicolumn{1}{c}{ P3 } & P4 & P5 \\
\hline \multirow{2}{*}{ Alg 1 } & mean $\left\|e_{k}\right\|$ & 54.38 & 47.21 & 43.45 & 65.23 & 48.63 \\
& min $\left\|e_{k}\right\|$ & 26.34 & 25.67 & 21.88 & 27.43 & 12.74 \\
\hline \multirow{2}{*}{ Alg 3 } & mean $\left\|e_{k}\right\|$ & 109.32 & 112.54 & 108.04 & 123.34 & 92.61 \\
& min $\left\|e_{k}\right\|$ & 67.10 & 72.98 & 62.49 & 79.01 & 53.89 \\
\hline \hline \multirow{2}{*}{ Alg 2 } & mean $\left\|e_{k}^{\Phi}\right\|$ & 4.86 & 5.11 & 4.70 & 7.57 & 4.72 \\
\cline { 2 - 7 } & min $\left\|e_{k}^{\Phi}\right\|$ & 0.67 & 1.23 & 1.19 & 2.48 & 1.85 \\
\hline
\end{tabular}

tracking error norms for participant P5 are shown in Fig. 3. Similarly, representative output signals for final trials are shown in Fig. 4. From the results collected it can be concluded that:

- The proposed data-driven gradient ILC for nonlinear system (Algorithm 1, shown by black line in figures) converges but is relatively slow. The convergence is not generally monotonic due to iterationvarying disturbances. However the effect of fatigue is compensated by the controller.

- The proposed data-driven point-to-point gradient ILC for nonlinear system (Algorithm 2, shown by the blue line in the figures) has far faster convergence, reflecting the theoretical property established in Theorem 4 that the convergence rate increases as point-to-point locations are removed. As previously noted, the error norm for Algorithm 2 is much smaller than the others as it only contains 5 points.

- The existing data-driven gradient ILC for linear systems (Algorithm 3, shown by the red dash line in the figures) cannot converge to a low error, which reflects the fact that the stimulated elbow rehabilitation system has significant nonlinearity. The tracking of Algorithm 3 in Fig. 4 may appear reasonable, however this level of accuracy is not satisfactory for the current application where high precision is required to maximise rehabilitation outcomes.

These results therefore confirm the ability of the new data-driven ILC algorithms to provide effective assistance of stroke patients' movement within a clinically relevant setting.

\section{Conclusions}

In this paper, a data-driven gradient ILC algorithm is developed for a general class of nonlinear systems. Precise tracking control is realized by the proposed algorithm without knowledge of the system dynamics. To achieve this goal, two additional attempts between each trial are required compared to the standard case where 


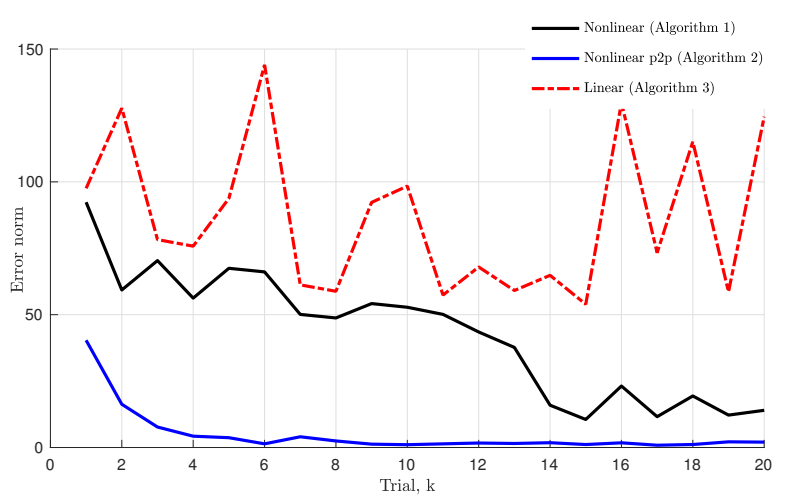

Fig. 3 Error norm convergence results of the three algorithms for participant P5.

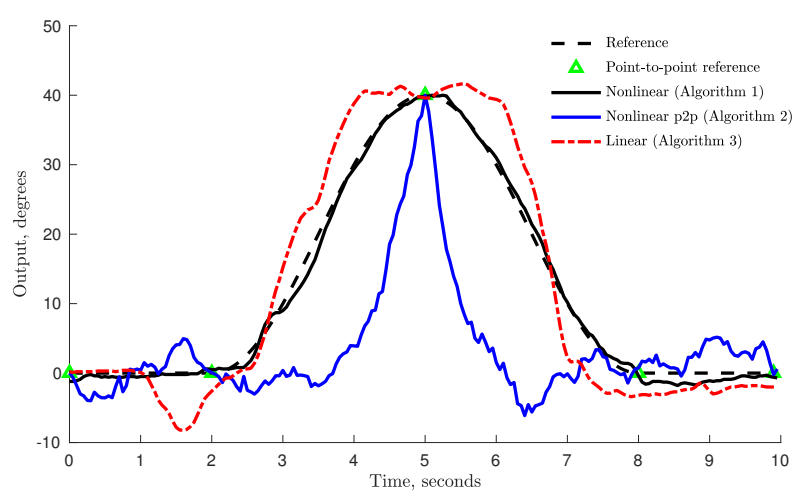

Fig. 4 Final trial of data-driven gradient ILC for linear systems (Algorithm 3) and nonlinear systems (Algorithm 1,2) for participant P5.

the model dynamics are known and no additional attempts are used. Each additional attempt is generated according to the tracking error of the last trial. Theoretical results confirm it monotonically converges to zero error. The algorithm is further extended to provide tracking control only at isolated points in the trajectory, thereby increasing convergence speed while still supporting the objective of many processes. The datadriven algorithms are experimentally evaluated by applying them to upper limb rehabilitation using electrical muscle stimulation. Results confirm they achieve high performance while avoiding the need for model identification which is impractical in a clinical setting. The approaches in this paper naturally extend to $m$-input, $p$-output MIMO systems, but require $m \times p$ intermediate trials within step 3 of both Algorithms 1 and 2 .

Future work will consider self-adaptive learning gain selection using input and output data. This will avoid the need for manual tuning and is expected to improve the convergence rate.

\section{Compliance with ethical standards}

\section{Conflict of interest}

The authors declare that they have no conflict of interest.

\section{Research involving Human Participants}

The study is part of a project named 'A feasibility study using iterative learning control to improve tracking accuracy of functional tasks' which has been approved by the Faculty of Engineering and Physical Sciences Ethics Committee (FEPS) of University of Southampton, UK. The project is registered on Ethics and Research Governance Online (ERGO) and the Ref number is 47701. Following this protocol, five healthy participants were recruited in this study.

\section{Informed consent}

Informed consent was obtained from all individual participants included in the study.

\section{Appendix}

\section{A. Proof of Proposition 1}

Proof Using equation (16), $\overbrace{\nabla \boldsymbol{g}\left(\hat{\boldsymbol{u}}_{k}\right) \tilde{\boldsymbol{e}}_{k}}=$

\begin{tabular}{|c|c|c|c|}
\hline $\begin{array}{c}\hat{D}_{k} \\
\hat{C} \hat{B}\end{array}$ & $\begin{array}{c}0 \\
\hat{D}\end{array}$ & $\begin{array}{ll}\cdots & 0 \\
\cdots & 0\end{array}$ & {$\left[\begin{array}{c}e_{k}(N-1) \\
\left.e_{k}-?\right)\end{array}\right.$} \\
\hline$C_{k} B_{k}$ & $D_{k}$ & $\cdots \quad 0$ & $e_{k}(N-2)$ \\
\hline$\hat{C}_{k} \hat{A}_{k}^{N-2}$ & & $\cdots \hat{D}_{k}$ & $e_{k}(0)$ \\
\hline
\end{tabular}

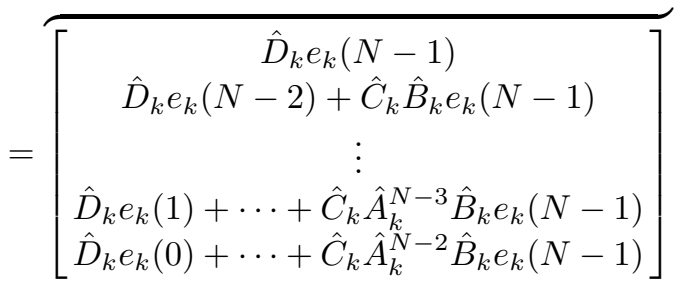




$$
\begin{gathered}
=\left[\begin{array}{c}
\hat{D}_{k} e_{k}(0)+\cdots+\hat{C}_{k} A^{N-2} \hat{B}_{k} e_{k}(N-1) \\
\hat{D}_{k} e_{k}(1)+\cdots+\hat{C}_{k} \hat{A}_{k}^{N-3} \hat{B}_{k} e_{k}(N-1) \\
\vdots \\
\hat{D}_{k} e_{k}(N-2)+\hat{C}_{k} \hat{B}_{k} e_{k}(N-1) \\
\hat{D}_{k} e_{k}(N-1)
\end{array}\right] \\
=\left[\begin{array}{cccc}
\hat{D}_{k} \hat{C}_{k} \hat{B}_{k} \cdots \hat{C}_{k} \hat{A}_{k}^{N-2} \hat{B}_{k} \\
0 & \hat{D}_{k} & \cdots & \hat{C}_{k} \hat{A}_{k}^{N-3} \hat{B}_{k} \\
\vdots & \vdots & \ddots & \vdots \\
0 & 0 & \cdots & \hat{D}_{k}
\end{array}\right]\left[\begin{array}{c}
e_{k}(0) \\
e_{k}(1) \\
\vdots \\
e_{k}(N-1)
\end{array}\right]
\end{gathered}
$$

$=\nabla \boldsymbol{g}\left(\hat{\boldsymbol{u}}_{k}\right)^{\top} \boldsymbol{e}_{k}$

Further, employing the properties of the time reversal operator and combining (58) with (18), we see

$$
\begin{aligned}
\nabla \boldsymbol{g}\left(\hat{\boldsymbol{u}}_{k}\right)^{\top} \boldsymbol{e}_{k} & =\overbrace{\frac{1}{\alpha}\left(\boldsymbol{g}\left(\hat{\boldsymbol{u}}_{k}+\alpha \tilde{\boldsymbol{e}}_{k}\right)-\boldsymbol{g}\left(\hat{\boldsymbol{u}}_{k}\right)\right)} \\
& =\frac{1}{\alpha} \overparen{\left(\boldsymbol{g}\left(\hat{\boldsymbol{u}}_{k}+\alpha \tilde{\boldsymbol{e}}_{k}\right)-\boldsymbol{g}\left(\hat{\boldsymbol{u}}_{k}\right)\right)} \\
& =\frac{1}{\alpha}\left(\overparen{\boldsymbol{g}\left(\hat{\boldsymbol{u}}_{k}+\alpha \tilde{\boldsymbol{e}}_{k}\right)}-\overparen{\boldsymbol{g}\left(\hat{\boldsymbol{u}}_{k}\right)}\right)
\end{aligned}
$$

Thus, the gradient ILC update law (20) is obtained.

\section{References}

1. Amann, N., Owens, D.H., Rogers, E.: Iterative learning control using optimal feedback and feedforward actions. International Journal of Control 65(2), 277-293 (1996)

2. An, J.Q., You, F.Y., Wu, M., She, J.H.: Iterative learning control for nonlinear weighing and feeding process. Mathematical Problems in Engineering (2018)

3. Arimoto, S., Miyazaki, F., Kawamura, S.: Bettering operation of dynamical systems by learning: a new control theory for servomechanism or mechatronics systems. In: Proceedings of the 23rd Conference on Decision and Control, pp. 1064-1069 (1984)

4. Barrreca, S., Wolf, S.L., Fasoli, S., Bohannon, R.: Treatment interventions for the paretic upper limb of stroke survivors: A critical review. Neurorehabilitation and Neural Repair 17(4), 220-226 (2003)

5. Barton, K.L., Alleyne, A.G.: A norm optimal approach to time-varying ILC with application to a multi-axis robotic testbed. IEEE Transactions on Control Systems Technology 19(1), 166-180 (2011)

6. Bolder, J., Kleinendors, S., Oomen, T.: Data-driven multivariable ILC: enhanced performance byeliminating L and Q filters. International Journal of Robust and Nonlinear Control 28, 3728-3751 (2018)

7. Boudjedir, C.E., Boukhetala, D., Bouri, M.: Iterative learning control of multivariable uncertain nonlinear systems with nonrepetitive trajectory. Nonlinear Dynamics 95(3), 2197-2208 (2019)

8. Bristow, D.A., Tharayil, M., Alleyne, A.G.: A survey of iterative learning control a learning-based method for high-performance tracking control. IEEE Control Systems Magazine 26(3), 96-114 (2006)

9. Buchheit, K., Pandit, M., Befort, M.: Optimal iterative learning control of an extrusion plant. In: International Conference on Control, vol. 1, pp. 652-657 (1994)
10. Butcher, M., Karimi, A., Longchamp, R.: Iterative learning control based on stochastic approximation. In: IFAC Triennial World Congress, vol. 17, pp. 1478-1483 (2008)

11. Chen, Y.Y., Chu, B., Freeman, C.T.: A coordinate descent approach to optimal tracking time allocation in point-to-point ilc. Mechatronics 59, 25-34 (2019)

12. Chi, R., Hou, Z., Jin, S., Huang, B.: An improved datadriven point-to-point ilc using additional on-line control inputs with experimental verification. IEEE Transactions on Systems, Man, and Cybernetics: Systems 49(4), 687$696(2019)$

13. Chien, C.J.: A combined adaptive law for fuzzy iterative learning control of nonlinear systems with varying control tasks. Ieee Transactions on Fuzzy Systems 16(1), 40-51 (2008)

14. Chu, B., Freeman, C.T., Owens, D.H.: A novel design framework for point-to-point ilc using successive projection. EEE Transactions on Control Systems Technology 23(3), 1156-1163 (2015)

15. Dinh Van, T., Freeman, C., Lewin, P.: Assessment of gradient-based iterative learning controllers using a multivariable test facility with varying interaction. Control Engineering Practice 29, 158-173 (2014)

16. Eraifej, J., Clark, W., France, B., Desando, S., Moore, D.: Effectiveness of upper limb functional electrical stimulation after stroke for the improvement of activities of daily living and motor function: a systematic review and meta-analysis. Systematic Reviews 6, 40 (2017)

17. Freeman, C.T.: Experimental evaluation of iterative learning control on a non-minimum phase plant. Ph.D. thesis, School of Electronics and Computer Science, University of Southampton (2004)

18. Freeman, C.T.: Upper limb electrical stimulation using input-output linearization and iterative learning control. IEEE Transactions on Control Systems Technology 23(4), 1546-1554 (2015)

19. Freeman, C.T., Cai, Z., Rogers, E., Lewin, P.L.: Iterative learning control for multiple point-to-point tracking application. IEEE Transactions on Control Systems Technology 99, 1-11 (2010)

20. Freeman, C.T., Hughes, A.M., Burridge, J.H., Chappell, P.H., Lewin, P.L., Rogers, E.: A model of the upper extremity using FES for stroke rehabilitation. ASME Journal of Biomechanical Engineering 131(3), 0310061-031006-10 (2009)

21. Hughes, A.M., Freeman, C.T., Burridge, J.H., Chappell, P.H., Lewin, P., Rogers, E.: Feasibility of iterative learning control mediated by functional electrical stimulation for reaching after stroke. Journal of Neurorehabilitation and Neural Repair 23(6), 559-568 (2009)

22. Huo, B., Freeman, C., Liu, Y.: Model-free gradient iterative learning control for non-linear systems. In: IFACPapersOnLine, vol. 52, pp. 304-309 (2019)

23. Janssens, P., Pipeleers, G., Swevers, J.: A datadriven constrained norm-optimal iterative learning control framework for lti systems. IEEE Transactions on Control Systems Technology 21(2), 546-551 (2013)

24. Lin, T., Owens, D.H., Hatonen, J.J.: Newton method based iterative learning control for discrete non-linear systems. International Journal of Control 79(10), 12631276 (2006)

25. Liu, T., Wang, D., Chi, R.: Neural network based terminal iterative learning control for uncertain nonlinear nonaffine systems. International Journal of Adaptive Control and Signal Processing 29(10), 1274-1286 (2015)

26. Liuzzo, S., Marino, R., Tomei, P.: Adaptive learning control of nonlinear systems by output error feedback. Ieee 
Transactions on Automatic Control 52(7), 1232-1248 (2007)

27. Meadmore, K.L., Exell, T., Hallewell, E., Hughes, A.M., Freeman, C.T., Kutlu, M., Benson, V., Rogers, E., Burridge, J.H.: The application of precisely controlled functional electrical stimulation to the shoulder, elbow and wrist for upper limb stroke rehabilitation: A feasibility study. Journal of NeuroEngineering and Rehabilitation 11, 105 (2014)

28. Meadmore, K.L., Hughes, A.M., Freeman, C.T., Cai, Z., Tong, D., Burridge, J.H., Rogers, E.: Functional electrical stimulation mediated by iterative learning control and $3 d$ robotics reduces motor impairment in chronic stroke. Journal of Neuroengineering and Rehabilitation 32(9), 1-11 (2012)

29. Moore, K.L.: Iterative Learning Control for Deterministic Systems. Springer-Verlag (1993)

30. Owens, D.H.: Iterative Learning Control: An Optimization Paradigm. Springer-Verlag London (2016)

31. Owens, D.H., Feng, K.: Parameter optimization in iterative learning control. International Journal of Control 76(11), 1059-1069 (2003)

32. Owens, D.H., Freeman, C.T., Chu, B.: An inverse model approach to multivariable norm optimal iterative learning control with auxiliary optimization. International Journal of Control 87(8), 1646-1671 (2014)

33. Owens, D.H., Freeman, C.T., Chu, B.: Generalized norm optimal iterative learning control with intermediate point and sub-interval tracking. International Journal of $\mathrm{Au}-$ tomation and Computing 12(3), 243-253 (2015)

34. Owens, D.H., Hätönen, J.J., Daley, S.: Robust monotone gradient-based discrete-time iterative learning control. International Journal of Robust and Nonlinear Control 19, 634-661 (2009)

35. Ratcliffe, J.D., Lewin, P.L., Rogers, E., Hatonen, J.J., Owens, D.: Norm-optimal iterative learning control applied to gantry robots for automation applications. IEEE Transactions on Robotics 22(6), 1303-1307 (2006)

36. Rogers, E., Owens, D.H., Werner, H., Freeman, C.T., Lewin, P.L., Kichhoff, S., Chmidt, S., Lichtenberg, G.: Norm optimal iterative learning control with application to problems in accelerator based free electron lasers and rehabilitation robotics. European Journal of Control 16(5), 497-524 (2010)

37. Rushton, D.N.: Functional electrical stimulation and rehabilitation - an hypothesis. Medical Engineering and Physics 25(1), 75-78 (2003)

38. Sampson, P., Freeman, C.T., Coote, S., Demain, S., Feys, P., Meadmore, K.L., Hughes, A.M.: Using functional electrical stimulation mediated by iterative learning control and robotics to improve arm movement for people with multiple sclerosis. IEEE Transactions on Neural Systems and Rehabilitation Engineering 24(2), 235-48 (2016)

39. Schindele, D., Aschemann, H.: ILC for a fast linear axis driven by pneumatic muscle actuators. In: IEEE International Conference on Mechatronics, pp. 967-972 (2011)

40. Tao, H.f., Paszke, W., Rogers, E., Galkowski, K., Yang, H.z.: Modified newton method based iterative learning control design for discrete nonlinear systems with constraints. Systems \& Control Letters 118, 35-43 (2018) 